\title{
Simple tests of renal function using creatinine, phenolsulphonphthalein, and pitressin
}

\author{
J. K. HEALY, K. D. G. EDWARDS, AND H. M. WHYTE \\ From the Medical Research Department, Kanematsu Memorial Institute, \\ Sydney Hospital, Sydney, Australia
}

SYNOPSIS The reciprocals of the blood urea nitrogen and plasma creatinine levels, the maximum specific gravity of the urine after vasopressin, and three modified forms of the phenolsulphonphthalein (P.S.P.) excretion test were found to be directly related to the glomerular filtration rate (G.F.R.) in hospital patients. From 34 to 75 patients were studied for each test, and in 21 patients all tests were performed concurrently.

The plasma creatinine level and the 15-minute urinary excretion of P.S.P. were found to be the most useful simple tests of renal function and gave sufficiently accurate estimates of total function (G.F.R.) to justify their more extensive use. The G.F.R. (ml./min./1.73 sq. m. of body surface area) could be calculated from each test, using the following equations:-

$$
\begin{aligned}
\text { G.F.R. } & =\frac{96 \cdot 8}{\text { plasma creatinine }}-4 \text { (males) } \\
& \text { or } \frac{60 \cdot 2}{\text { plasma creatinine }}+12 \text { (females), and }
\end{aligned}
$$

G.F.R. $=3.15 \times$ P.S.P. $\%+19$, where P.S.P. $\%$ is the 15 -minute urinary excretion of P.S.P., expressed as a percentage of the administered dose. Satisfactory estimates of G.F.R. were also given

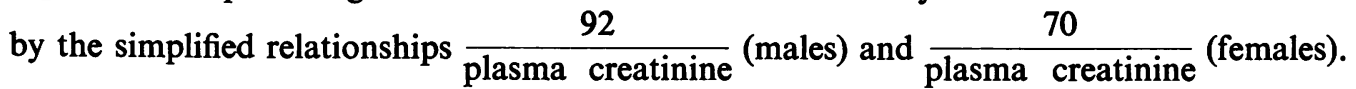

In the presence of impaired renal function more accurate estimates of G.F.R. were obtained from the plasma creatinine and P.S.P. excretion tests (S.D. of estimate 8 and $13 \mathrm{ml} . / \mathrm{min}$. respectively). It was thought that these tests could well replace the commonly used blood urea nitrogen estimation as simple tests of renal function.

It has generally been regarded that renal function consists of two relatively distinct aspects, glomerular filtration and tubular activity, for each of which tests of varying degrees of complexity have been devised. However, there is a growing recognition that in the majority of renal diseases both aspects suffer more or less equally and that a measure of glomerular filtration rate (G.F.R.) alone provides a reasonable assessment of the total renal functional mass. The most accurate methods for measuring G.F.R. are the inulin clearance and the endogenous creatinine clearance, which are equally reliable provided errors due to false chromogens in the creatinine estimation are eliminated (Haugen and Blegen, 1953; Edwards, 1959a). Creatinine clearance can be measured with less trouble than the inulin Received for publication 16 December 1963. clearance, and has proved valuable in assessing the progress of renal failure (Edwards, 1959b; Edwards and Whyte, 1959a; Wrong, 1962). Even so, it is a relatively complex and inconvenient procedure, and there is a need for simpler methods for measuring renal function in clinical practice. In this regard, the plasma creatinine level, the blood urea nitrogen level, the urea clearance, and the maximum urinary specific gravity achieved after water deprivation have been examined previously (Edwards and Whyte, 1959b; Castaldi, Edwards, and Whyte, 1960) using the G.F.R. estimated from creatinine clearance as a yardstick of renal function. It was concluded that the plasma creatinine level was a more sensitive indicator of renal function than the blood urea level if males and females were treated separately and muscle wasting was not present, and that G.F.R. 
could be predicted reasonably well from the specific gravity test in generalized renal disease. Doolan, Alpen, and Theil (1962) came to similar conclusions with regard to plasma creatinine and creatinine chromogen levels as tests of renal function in naval personnel, but advised the measurement of full creatinine clearance wherever possible.

The present report confirms and extends the previous work and gives an assessment of four additional tests, namely, the maximum urinary specific gravity produced after an injection of vasopressin, the plasma level of phenolsulphonphthalein (phenol red, P.S.P.) 30 minutes after its intravenous injection, and the urinary excretion of this dye in 15 minutes and two hours. The most satisfactory of these tests proved to be the 15minute excretion of phenolsulphonphthalein. The G.F.R. could be predicted with reasonable accuracy from the results of the test and, being simple and rapid, it seems that the test deserves greater recognition.

Rowntree and Geraghty (1910) were the first to suggest the use of P.S.P. for testing renal function. They recommended an intramuscular injection of $1 \mathrm{ml}$. (6 mg.) for all patients, regardless of body weight or size, and found that 40 to $60 \%$ was excreted in the urine within one hour and a further 20 to $25 \%$ in the second hour by normal subjects. Chapman and Halsted (1933) suggested a 15minute urinary collection time, but apart from this and the occasional suggestion that the dye should be given intravenously there has been no change in the test for 53 years. Lapides and Bobbitt (1958) compared the results of the 15-minute excretion test with urea and creatinine clearances but made no statistical analysis of their data. Their principal findings were that a normal P.S.P. excretion was always associated with a normal G.F.R. but poor excretion of dye was not necessarily indicative of an impaired glomerular filtration rate.

The popularity of the standard P.S.P. test has not extended to any degree beyond the United States of America, perhaps because it is regarded only as a crude measure of tubular function. In the present study we have modified the test to provide for individual variations of plasma volume by adjusting dosage to surface area (Edwards and Whyte, 1960), and have made a critical assessment of its relation to the glomerular filtration rate.

\section{MATERIAL AND METHODS}

The 75 patients were admitted to the Medical Research Ward, Sydney Hospital, for treatment of anuria or for investigation of renal function. Thirteen were in the diuretic phase of 'acute tubular necrosis' (five with superadded renal infection); 11 were recovering fromô decompensation of chronic renal failure due to chronic pyelonephritis; six were recovering from acute glomerulo $\overrightarrow{\vec{s}}$ nephritis; 15 had compensated chronic renal failureassociated with hypertensive cardiovascular disease (5), chronic pyelonephritis (5), chronic glomerulonephritis (4), and polycystic disease (1); 14 had essential hypertension without renal failure; 14 were 'normals' (hospital 2 patients without evidence of cardiovascular, renal, or electrolyte disorders); and only two had renal medullary syndromes without impaired glomerular function (renal tubular acidosis, medullary nephrocalcinosis); there wereno cases of acute pyelonephritis, and none with recurrent pyelonephritis in the absence of chronic renal failure.

CREATININE CLEARANCE AND PLASMA CREATININE LEVEL $\vec{\nabla}$ The methods have been described fully elsewherecir (Edwards and Whyte, 1958, 1959b). The patient was given G no tea, coffee, meat, fish, or drugs in the 12 hours $V$ preceding the test. Over a preliminary period of two응 hours $750 \mathrm{ml}$. of water was given and then $200 \mathrm{ml}$. wasgiven each hour for six hours during which two accuratelycs timed three-hour collections of urine were made. $A_{0}^{\mathbb{D}}$ heparinized blood sample was collected at some timed during the test.

Creatinine was measured in plasma and urine byळ్ separating it from other chromogens by adsorption on to $\rightarrow$ fuller's earth, with subsequent elution of creatinine and the development of colour with alkaline picrate. Protein. ${ }^{+}$ precipitation, $p \mathrm{H}$, and temperature were controlled. The endogenous creatinine clearance was corrected to surface area of 1.73 square metres.

UREA NITROGEN Urea nitrogen in blood and urine was measured in the Biochemistry Department by an auto- $\varrho$ mated diacetyl tec.nnique (AutoAnalyzer method 2).

THE VASOPRESSIN TEST Without special preparation of the patient, $1 \mathrm{ml}$. (5 units) of vasopressin tannate in oilo (Pitressin) was administered intramuscularly at 7 a.m The ampoule was warmed and shaken to ensure that the hormone was dissolved. The specific gravity of the urineo passed at 10 a.m., 1 p.m., and 4 p.m. was measured and 3 . corrected for temperature and gross proteinuria. Glyco-i̊ suria was not encountered. The maximum specific -3 gravity attained was recorded (de Wardener, 1956).

P.S.P. TESTS These measured the level of the dye in plasma and the concentration of P.S.P. in urine.

(a) Plasma level Previous studies (Healy, 1959, N unpublished observations) showed that the plasma? concentrations of the dye fell rapidly after an intravenous 0 injection in 13 patients with normal renal function and more slowly in 30 patients with varying degrees of renal impairment. The discrepancies were well established by< 30 minutes and, with the dosage employed, plasma levels, were still high enough at this time to be accurately $\bar{D}$ measured. Therefore the plasma level at 30 minutes was ${ }^{+}$ selected for examination as an index of renal function in a larger series of patients.

The patients were fasted overnight and kept recumben $\mathbb{D}$ throughout the test. A water load of $500 \mathrm{ml}$. was given in 
the half-hour preceding the injection. After withdrawing $10 \mathrm{ml}$. of blood for a plasma blank a dose of $3 \mathrm{ml}$. (18 mg.) of P.S.P. per square metre of body surface area was injected through the same needle. Blood was drawn into the syringe and mixed with the dye and the injection completed in one minute. At 30 minutes a second sample of heparinized blood was collected. A modification of the method of Ochwadt and Pitts (1956) was used for estimating the plasma level of phenolsulphonphthalein. Four ml. of plasma from each sample was pipetted into large, glass-stoppered contrifuge tubes with $10 \mathrm{ml}$. of acetone, $2 \mathrm{ml}$. of concentrated detergent (Teepol), and 3 drops of $50 \%$ sodium hydroxide. The tubes were then vigorously shaken. Plasma proteins and any free haemoglobin were precipitated by the acetone and residual turbidity was removed by the detergent. The tubes were then centrifuged at 3,000 r.p.m. for five minutes. The clear supernatant, coloured pink in the specimen containing the P.S.P., was poured into cells with a $4 \mathrm{~cm}$. light path, covered, and the optical density measured in a Unicam spectrophotometer at a wavelength of $560 \mathrm{~m} \mu$. The concentration of P.S.P. in milligrams per $100 \mathrm{ml}$. was determined by reference to a previously prepared calibration curve for known concentrations of P.S.P. added to pooled human plasma.

(b) The urine tests The same ward procedure was used as in $(a)$ but when the plasma level of dye was not being measured fasting was not necessary. The bladder was emptied and all urine collected at exactly 15 minutes and again at two hours after the injection of dye. The concentration of P.S.P. in the urine was measured by pipetting 1 volume of urine and 1 volume of $50 \%$ sodium hydroxide into a volumetric flask and making it up to 20 volumes with water. Occasionally the solution was not water clear, in which case it was centrifuged at 3,000 r.p.m. for five minutes. The optical density of the reddishpurple solution was then read in a $1 \mathrm{~cm}$. cell in a Unicam spectrophotometer at a wavelength of $560 \mathrm{~m} \mu$. The reading was converted to P.S.P. concentration by reference to a previously prepared calibration curve. The total volumes of the 15-minute and two-hour urine collections were measured and the quantities of P.S.P. in each expressed as a percentage of the injected dose. The calibration curves for both the urine and plasma P.S.P. standards were linear.
Results were analysed statistically according to Moroney (1956) and Quenouille (1952).

\section{RESULTS}

The first group studied comprised 21 patients each of whom had been submitted to all six tests and a creatinine clearance estimation within a period of three days. The regression equations for the best fitting straight line relationships between G.F.R. and the results obtained in each test, together with the standard deviation of the estimates, are given in Table I. It was necessary to use reciprocal values for blood urea nitrogen and plasma creatinine levels to produce the best linear relationships; the raw data and logarithmic derivatives proved less useful. For the vasopressin test the last two digits of the recorded specific gravity were used. The results indicate that G.F.R. could best be predicted from the plasma creatinine level reciprocal and the 15-minute urinary excretion of phenolsulphonphthalein. The measurement of the plasma level of P.S.P. and urinary excretion of the dye in two hours offered no advantages over the 15-minute P.S.P. test. The post-vasopressin specific gravity was moderately useful for the prediction of G.F.R., and all these tests were preferable to the measurement of blood urea.

In larger groups of patients, 34 to 75 in number, comparisons were made between the G.F.R. and one or more of the other tests. The results, given in Table II, are similar to those in the first group. A significant sex difference was found in the G.F.R.plasma creatinine relationship, confirming previous work (Edwards and Whyte, 1959b), but not in the other comparisons. In addition to the full regression equations, Table II shows the simplified relationships between G.F.R. and plasma creatinine which are used in our laboratory and have proved to be satisfactory for routine use. The predictive value of

TABLE I

COMPARISON OF VARIOUS SIMPLE TESTS OF RENAL FUNCTION WITH GLOMERULAR FILTRATION RATE (G.F.R.) IN 21 PATIENTS SUBJECTED TO ALL TESTS

Test Used for Prediction of G.F.R. Regression Equations for G.F.R. (ml./min.)
Urinary P.S.P. excretion in 15 minutes ( $\%$ of dose)

Urinary P.S.P. excretion in 120 minutes ( $\%$ of dose)

Plasma P.S.P. concentration at 30 minutes $(\mathrm{mg} . / 100 \mathrm{ml}$.)

Maximum urinary S.G. after vasopressin (last two digits only)

Blood urea $\mathrm{N}$ concentration ( $\mathrm{mg} . / 100 \mathrm{ml}$.)

Separate equations for males (M) and females (F) are necessary for estimation of G.F.R. from plasma creatinine concentration.

$\begin{array}{ll}\text { (M) G.F.R. }=\frac{132.3}{P_{C R}}-20 & \pm 17 \\ \text { (F) G.F.R. }=\frac{57 \cdot 1}{P_{C R}}+17 & \\ \text { G.F.R. }=2.9 \times \text { P.S.P. } \%+17 & \pm 20 \\ \text { G.F.R. }=1.5 \times \text { P.S.P. } \% \frac{1}{\text { G.F.R. }=191-1,160 \times P_{\text {P.S.P. }}} & \pm 21 \\ \text { G.F.R. }=6.49 \times \text { S.G. }-20 & \pm 25 \\ \text { G.F.R. }=\frac{1,006}{\text { B.U.N. }}+21.5 & \pm 29\end{array}$


TABLE II

COMPARISON OF VARIOUS SIMPLE TESTS OF RENAL FUNCTION WITH GLOMERULAR FILTRATION RATE (G.F.R.) IN LARGER NUMBERS OF HOSPITAL PATIENTS

Test Used for Prediction of G.F.R.

No. of Regression Equations for G.F.R. Patients (ml./min.)

Urinary P.S.P. excretion in 15 minutes (\% of dose)

Blood urea $\mathrm{N}$ concentration $(\mathrm{mg} . / 100 \mathrm{ml}$.)

Maximal endogenous urea clearance ( $\%$ of normal)

Plasma P.S.P. concentration at 30 minutes $(\mathrm{mg} . / 100 \mathrm{ml}$.)

Urinary P.S.P. excretion in 120 minutes ( $\%$ of dose)

Maximum urinary S.G. after vasopressin (last two digits only)

Plasma creatinine concentration (mg./100 ml.) $)^{1}$

$\begin{array}{lll}75 & \text { (M) G.F.R. }=\frac{96.8}{P_{C R}}-4 & \pm 19 \\ (42 \mathrm{M}, & & \\ 33 F) & \text { (F) G.F.R. }=\frac{60.2}{P_{C R}}+12 & \\ 75 & \text { G.F.R. }=3.15 \times \text { P.S.P. } \%+19 & \pm 23 \\ 75 & \text { G.F.R. }=\frac{1,180}{\text { B.U.N. }}+14 & \pm 30 \\ 34 & \text { G.F.R. }=1.43 \times C_{U}+11 & \pm 19 \\ 40 & \text { G.F.R. }=190-1,120 \times P_{P . S . P .} & \pm 24 \\ 40 & \text { G.F.R. }=1.43 \times \text { P.S.P. } \%+9 & \pm 26 \\ 39 & \text { G.F.R. }=5.83 \times \text { S.G. }-19 & \pm 29 \\ 136 & \text { (M) G.F.R. }=\frac{94.3}{P_{C R}}-2 & \pm 14 \\ & \text { (F) G.F.R. }=\frac{69.9}{P_{C R}}+2 & \\ 50 & \text { G.F.R. }=5.9 \times \text { S.G. }-33 & \end{array}$

Maximum urinary S.G. after water deprivation (last two digits only) ${ }^{\mathbf{1}}$ $\pm 19$ $\pm \mathbf{2 3}$ $\pm 30$

19

\pm 24
\pm 26

$+29$

Standard Deviation of Estimate of G.F.R.

Different equations for males (M) and females (F) are necessary in the case of creatinine. Simplified equations for estimating G.F.R. from creatinine level or 15-minute P.S.P. excretion are obtained from the following equations, which are used routinely for our laboratory reports:G.F.R. estimated from $P_{C R}=\frac{92}{P_{C R}}$ (males) or $\frac{70}{P_{C R}}$ (females), with S.D. $\pm 19 \mathrm{ml} . / \mathrm{min}$.;

G.F.R. estimated from 15-minute P.S.P. excretion $=4.6 \times$ P.S.P. $\%$ (males) or $3.7 \times$ P.S.P. $\%$ (females), with S.D. \pm 24 ml./min.

${ }^{1}$ Data taken from previous publications (Edwards and Whyte, 1959b; Castaldi et al., 1960).

TABLE III

ANALYSIS OF DATA FROM PATIENTS WITH IMPAIRED GLOMERULAR FILTRATION RATE (G.F.R. LESS THAN 90 ML./MIN./1.73 SQ. M.)

Test Used for Prediction of G.F.R.

No. of

Regression Equations for G.F.R.

Standard Deviation of the Estimate (ml./min.)

Plasma creatinine concentration (mg./100 ml.)

40

40

Urinary P.S.P. excretion in 15 minutes (\% of dose) Maximum urinary S.G. after vasopressin (last two digits only)

$\begin{array}{ll}\text { (M.) G.F.R. }=\frac{82.4}{P_{C R}}-1 & \pm 8 \\ \text { (F.) G.F.R. }=\frac{62.1}{P_{C R}}+3 & \\ \text { G.F.R. }=2.75 \times \text { P.S.P. } \%+13 & \pm 13 \\ \text { G.F.R. }=3.9 \times \text { S.G. }-3 & \pm 20\end{array}$

Separate equations for males (M) and females (F) are necessary for estimating G.F.R. from plasma creatinine concentration.

the tests was greater when the analysis was confined to patients with a G.F.R. of less than $90 \mathrm{ml}$. per minute per 1.73 square metres (Table III). Comparisons of the true G.F.R. (from the endogenous creatinine clearance) and the G.F.R. as predicted from the plasma creatinine level, the excretion of P.S.P. in the urine in 15 minutes, or the maximum urinary specific gravity after vasopressin are shown in Figures 1, 2, and 3.

\section{DISCUSSION}

The results show that the G.F.R. can be estimated reasonably well in most hospital patients by means of any one of a number of simple renal function tests. Whilst full clearance tests give the most accurate assessment of overall renal function, for many practical purposes such as diagnosing the presence or absence of renal disease, including pre-operative assessment, and following progress of established renal disease, a less precise and simpler method would often suffice. Probably the test employed most commonly in this regard is the measurement of $\frac{D}{O}$ blood urea but, as our results have shown, it only crudely reflects the glomerular filtration rate, $N$ largely due to the marked variation in protein $N$ consumption between patients which can only be overcome by doing full clearance studies (see Table $\omega$ II). The tests examined in this study proved to be superior to the blood urea nitrogen level.

THE PLASMA CREATININE LEVEL As an index of renal $\stackrel{\infty}{+}$ function this has the advantages of not requiring any urine collection and of only one venepuncture. No special preparation of the patient is needed $\frac{?}{9}$ beyond avoiding a creatinine-rich diet or excessive 


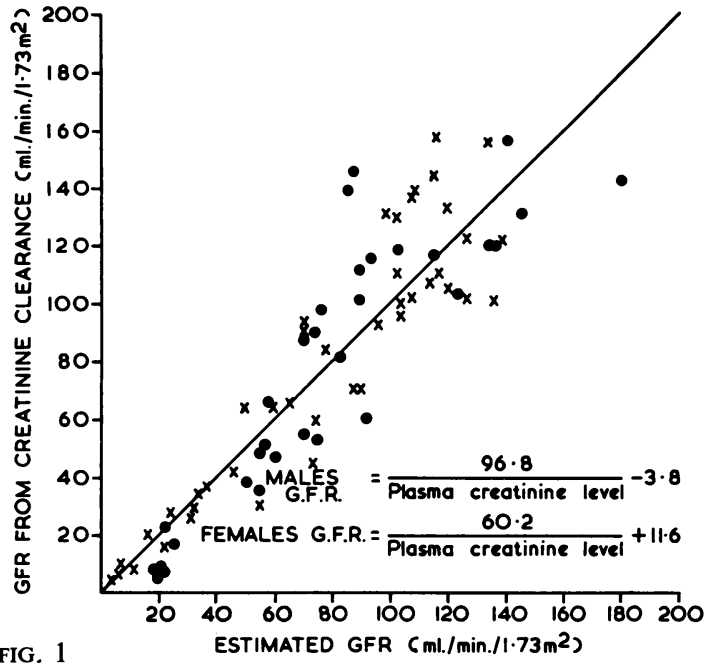

FIG. 1

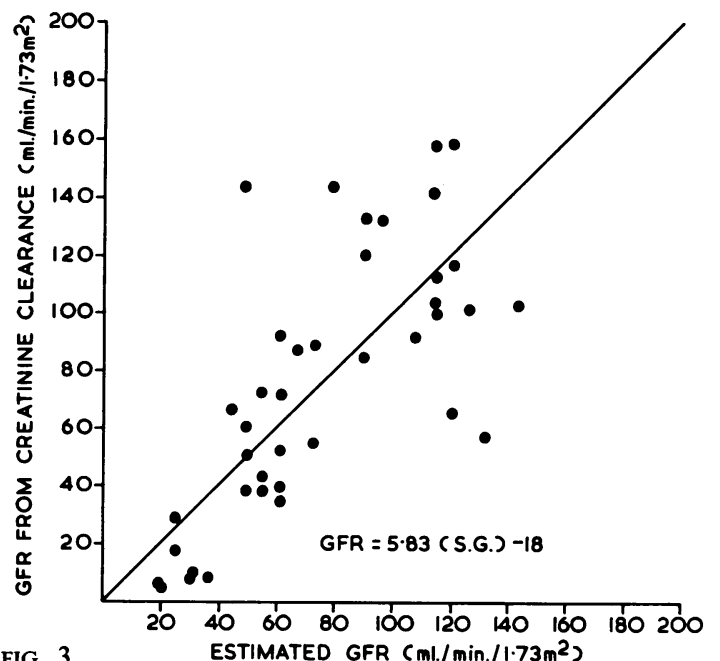

FIG. 3

exertion before the test. In the past, inaccurate methodology has limited the usefulness of the test (Milne, 1957), but techniques are now available with a 'standard error of the method' of $1.6 \%$ (Edwards and Whyte, 1958). With the advent of auto-analyzers, more extensive use of this test is becoming feasible (Chasson, Grady, and Stanley, 1961). Routine reporting of the estimated G.F.R., calculated separately for males and females, facilitates the interpretation of results. For this purpose, the simplified relationships of G.F.R. $=\frac{92}{\text { plasma creatinine }}$

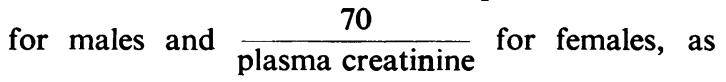

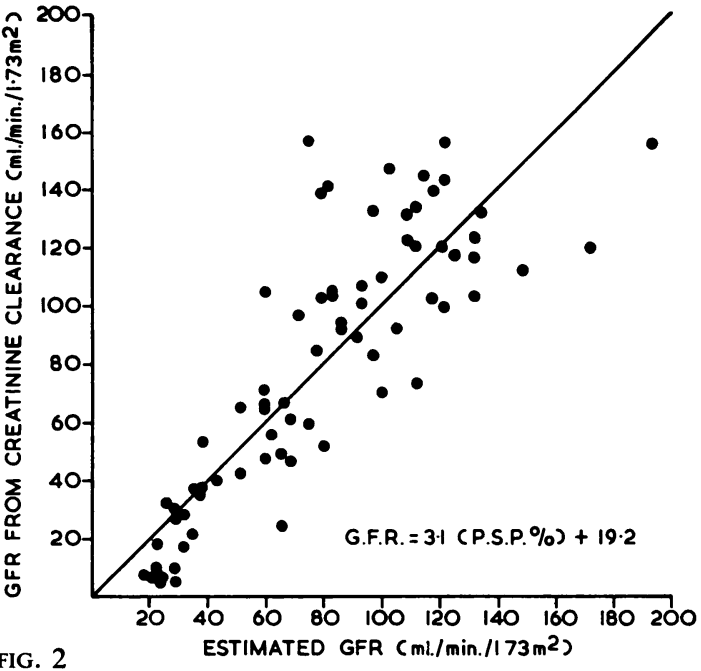

FIG. 1. The relationship between actual glomerular filtration rate and the glomerular filtration rate as estimated from the reciprocal of the plasma creatinine level (mg. per $100 \mathrm{ml}$.) for males $(X)$ and females $(0)$.

FIG. 2. The relationship between actual glomerular filtration rate and the glomerular filtration rate as estimated from the percentage of an injected dose of P.S.P. appearing in the urine within 15 minutes.

FIG. 3. The relationship between actual glomerular filtration rate and the glomerular filtration rate as estimated from the last two digits of the maximum urine specific gravity attained after the injection of vasopressin.

shown in Table II, are satisfactory. It is important to observe sex differences and reciprocal relationships in these equations, as stressed previously (Edwards and Whyte, 1959b), otherwise the test appears less useful (Wrong, 1962).

THE P.S.P. TESTS The 30-minute plasma level and the two-hour urinary excretion of P.S.P. can be seen from the results to offer no advantages over the 15-minute urinary excretion test (Tables 1 and II). They both take longer to perform and the 30minute plasma level test necessitates a second venepuncture although it avoids the disadvantage of urine collection. 
The modified 15-minute urinary P.S.P. test can be performed very rapidly in a modestly equipped laboratory. It is possible that it could be carried out satisfactorily but with some loss of precision with a simple colour comparator of the type advocated for haemoglobin estimations. In that case the test could readily be performed in the ward, the clinic, or in the doctor's surgery. The dye is cheap and although a small number of patients suffer allergic reactions, no serious toxicity has been ascribed to it in spite of long and extensive use. The test has the disadvantage of requiring complete emptying of the bladder at one specified time, but there is no necessity for the bladder to be emptied at the time of the injection. Incomplete emptying will lead to falsely low estimates of renal function, as with clearance procedures, and it is wise to ensure a good urinary flow.

The results indicate that the accuracy of the test as a means of predicting G.F.R. is increased where the G.F.R. is reduced and therefore it is of considerable value in following progress in known renal disease.

Phenolsulphonphthalein is actively secreted by the proximal tubules (Marshall and Vickers, 1923; Chambers and Kempton, 1933; Forster, 1948; Taggart and Forster, 1950; Young and Edwards, 1963) aided by an enzyme complex which is also involved in the transport of other substances (Beyer, Painter, and Wiebelhaus, 1950; Smith, 1951; Taggart, 1958; Edwards, Crawford, Dempster, Milne, and Sicinski, 1961; Edwards, 1962). As there is a maximal tubular excretory rate for P.S.P. which is exceeded when the plasma level rises much above $1 \mathrm{mg}$. per $100 \mathrm{ml}$. (Marshall and Crane, 1924; Shannon, 1935; Smith, Goldring, and Chasis, 1938), the dose of P.S.P. used in this work was selected to avoid exceeding this critical plasma concentration. It was felt that a dose given according to body surface area would allow for individual differences in plasma volume (Edwards and Whyte, 1960), and the intravenous route was chosen to avoid errors of absorption.

A number of patients were considered unsuitable for the P.S.P. tests in this work. Smith et al. (1938) demonstrated competitive inhibition reducing P.S.P. excretion when a number of other substances were present. These are now known to include penicillins, sulphonamides, salicylates, and diuretics (Taggart, 1958; Edwards, 1962) and for this reason these were not given in the 24 hours preceding the P.S.P. tests. Gouty subjects were also avoided in case tubular reabsorption of uric acid should compete with P.S.P. excretion. Also, Rowntree and Geraghty (1910) and Marshall and Vickers (1923) demonstrated some hepatic uptake and excretion of P.S.P. by mechanisms thought to be similar to those found in the renal tubules (Edwards, 1961), and for this reason patients with severe liver disease were not included in the present study. Again, since $75 \%$ of the dye is bound to albumin in the plasma (Marshall and Vickers, 1923; Grollman, 1925) and the excretion rate of P.S.P. could be affected by hypoalbuminaemia, this condition was excluded from the study. Myeloma protein was also found to bind the dye very firmly and patients with multiple myeloma were omitted. It is not yet known whether these objections significantly detract from the validity of the modified 15-minute urinary P.S.P. test, but it seemed that falsely low estimates of G.F.R. were obtained in some patients with gout or hyperuricaemia.

THE VASOPRESSIN TEST This has the advantage of avoiding timed complete urine collections. Its $\bar{c}$ principal disadvantage appears to be its relatively $\mathbb{D}$ poor correlation with the G.F.R., compared with $\mathbb{D}$ the results for the plasma creatinine and the 15minute urinary P.S.P. test. Also, it requires an $\stackrel{\mathbb{D}}{-}$ injection, sometimes painful, and may produce $\vec{\varphi}$ water intoxication in infants (Wrong, 1962) or $\stackrel{\curvearrowright}{\nexists}$ angina in patients with coronary artery disease (Goodman and Gilman, 1955). One reason for its loss of accuracy in predicting the G.F.R. may be the difficulty in dissolving the hormone completely and its variable period of action. Also, de Wardener (1956) showed that vasopressin did not produce as high a maximum specific gravity as did dehydration alone. Our experience confirmed this, with a difference of about 2 units of specific gravity. The dehydration test appears to offer a slightly better correlation with G.F.R. (Castaldi et al., 1960) and 종 does not require an injection, but it necessitates a $\dot{0}$ long and uncomfortable period of dehydration which can be dangerous in the presence of renal $\delta$ insufficiency. Also the cooperation of patients cannot always be depended upon in the dehydration $\mathrm{O}$ test. A small number of patients with specific renal medullary or collecting duct disorders were included o amongst the group and these would tend to upset the correlation; such disorders included the renal $/ \mathrm{N}$ tubular acidosis syndrome, either inherited or related N to hypokalaemia or hypercalcaemia (Wrong and Davies, 1959; Edwards, 1962). It is an interesting feature of all these tests that although they individually test different parts of the nephron, the majority seem to bear a linear relationship to the G.F.R. and therefore to each other. This supports the increasingly popular theory that most renal diseases affect, 0 and cause the loss of, whole nephrons rather than $\vec{\Phi}$ particular aspects of nephron function. Thus $\frac{\mathrm{Q}}{\mathbb{Q}}$ urinary concentrating ability, the excretion of $\frac{\varrho}{\sigma}$ 
creatinine, urea, and P.S.P., and even ammonia synthesis by the collecting ducts seem to show a linear relationship to G.F.R. in generalized renal disease (Wrong, 1962; Edwards, 1962).

We are grateful to Dr. D. W. Piper of the Royal North Shore Hospital who stimulated the preliminary studies on P.S.P. with assistance from the Biochemistry Department of that hospital. We are also indebted to Dr. F. C. Neale and the staff of the Biochemistry Department, Kanematsu Memorial Institute, for measurements of urea; to Miss Coralie Campbell for valuable technical assistance; and to the medical and nursing staff of the Clinical Research Ward, Sydney Hospital, for assistance with urine collection and vasopressin tests.

\section{REFERENCES}

Autoanalyzer Method 2 (1960). Technicon Instruments Corp., New York.

Beyer, K. H., Painter, R. H., and Wiebelhaus, V. D. (1950). Amer. J. Physiol., 161, 259.

Castaldi, P. A., Edwards, K. D. G., and Whyte, H. M. (1960). Med. J. Aust., 1, 847.

Chambers, R., and Kempton, R. G. (1933). J. cell. comp. Physiol., 3, 131.

Chapman, E. M., and Halsted, J. A. (1933). Amer. J. med. Sci., 186, 223.

Chasson, A. L., Grady, H. J., and Stanley, M. A. (1961). Amer. J. clin. Path, 35, 83.

De Wardener, H. E. (1956). Lancet, 1, 1037.

Doolan, P. D., Alpen, E. L., and Theil, G. B. (1962). Amer. J. Med., 32,65 .
Edwards, K. D. G. (1959a). M.D. Thesis, University of Sydney.

- (1959b). Aust. Ann. Med., 8, 195.

- (1961). Med. Res., 1, 13.

- (1962). Aust. Ann. Med., 11, 59.

—, Crawford, M. A., Dempster, W. J., Milne, M. D., and Sicinski, A. (1961). Clin. Sci., 21, 175.

-

- - (1959a). Med. J. Aust., 1, 417.

- (1959b). Aust. Ann. Med., 8, 218.

- - (1960). Clin. Sci., 19, 399.

Forster, R. P. (1948). Science, 108, 65

Goodman, L. S., and Gilman, A. (1955). The Pharmacological Basis of Therapeutics, 2nd ed. Macmillan, New York.

Grollman, A. (1925). J. biol. Chem., 64, 141.

Haugen, H. N., and Blegen, E. M. (1953). Scand. J. clin. Lab. Invest., $5,67$.

Lapides, J., and Bobbitt, J. M. (1958). J. Amer. med. Ass., 166, 866.

Marshall, E. K., Jr., and Crane, M. R. (1924). Amer. J. Physiol., 70,

—_, and Vickers, J. L. (1923). Bull. Johns Hopk. Hosp., 34, 1.

Milne, M. D. (1957). In Biochemical Disorders in Human Disease, edited by R. H. S. Thompson and E. J. King, p. 177. Churchill, London.

Moroney, M. J. (1956). Facts from Figures, 3rd ed. A236. Penguin Books.

Ochwadt, B. K and Pitts, R. F. (1956). Amer. J. Physiol., 187, 318.

Quenouille, M. H. (1952). Associated Measurements. Butterworth, London.

Rowntree, L. G., and Geraghty, J. T. (1910). J. Pharmacol. exp. Ther., $1,579$.

Shannon, J. A. (1935). Amer. J. Physiol., 113, 602.

Smith, H. W. (1951). The Kidney. Oxford University Press, London.

- Goldring, W., and Chasis, H. (1938). J. clin. Invest., 17, 263.

Taggart, J. V. (1958). Amer. J. Med., 24, 774.

, and Forster, R. P. (1950). Amer. J. Physiol., 161, 167.

Wrong, O. M. (1962). In Renal Disease, edited by D. A. K. Black, p. 440. Blackwell, Oxford.

- , and Davies, H. E. F. (1959). Quart. J. Med., 28, 259.

Young, J. A., and Edwards, K. D. G. (1963). Med. Res., 1, 83. 